\title{
Kongresse | Kurse
}

Berlin

3. -5.5 .2017$

World Congress Integrative Medicine \& Health 2017

$10^{\text {th }}$ ECIM \& 12th ICCMR Congress

www.ecim-iccmr.org/2017

\section{Bad Wörishofen}

8.-12.5.2017

Zusatzbezeichnung Naturheilverfahren

Start Kursblock B

www.kneippaerztebund.de

Patras/Griechenland

15.-17.5.2017

5th International Phytocosmetics and

Phytotherapy Congress

www.phytoessence.org/IPPC2017

\section{Stuttgart}

\subsubsection{7}

Interaktionen von Phytopharmaka und

Pflanzenextrakten mit allopathischer

Medikation

Apothekerin Margit Schlenk

Olga-Hospital, Hörsaal Olga-Raum 1;

Beginn 20 Uhr c.t. Die Veranstaltung ist von der

Landesapothekerkammer für die Punktefort-

bildung akkreditiert.

Deutsche Pharmazeutische Gesellschaft

www.dphg.de

Engelberg/Schweiz

18.-20.5.2017

Grundkurs Phytotherapie

Kurs 1 der SMGP-Ausbildung Phytotherapie www.smgp.ch

Rothenburg

23. -27.5 .2017$

48. TCM-Kongress

Shen und Emotionen, Herz-Kreislauf-Erkrankungen, Bian Que und die 4 diagnostischen Methoden www.tcm-kongress.de

Hannover

1.6.2017

Cannabis

Prof. Dr. Erich Schneider

20 Uhr s.t. im Hörsaal des ehemaligen Apothekengebäudes (Institut für Geschichte der Veterinärmedizin) der Tierärztlichen Hochschule. Der Vortrag wird mit 3 Fortbildungspunkten der Apothekerkammer Niedersachsen bewertet. Deutsche Pharmazeutische Gesellschaft www.dphg.de
Kiel

$$
\text { 27.-29.6.2017 }
$$

2nd International Conference of Marine Fungal Natural Products (MaFNaP)

www.geomar.de/en/research/fb3/fb3-mn/

symposia/mafnap2017

Pisa / Italien

28.-30.6.2017

XV National Congress joint with the 1st International Congress on Edible, Medicinal and Aromatic Plants (ICEMAP 2017)

http://users.unimi.it/phytosif/congresso.html

\section{Graz/Österreich}

2.-5.7.2017

Phytopharm 2017 and

10th Anniversary of the TCM Research

Center Graz

www.doclinika.ru/Phyto17/index.html

Hamburg

4.7.2017

Marine Biotechnologie - Wirkstoffe aus dem Meer

Prof. Dr. Thomas Schweder, Universität Greifswald 19.30 Uhr s.t. im Großen Hörsaal des Instituts für Pharmazie

Deutsche Pharmazeutische Gesellschaft e.V. www.dphg.de

Mainz

24-28.7.2017

International Conference on Science and Society 2017: "Phytomedicine and Biopiracy"

Chairman: Prof. Dr. Thomas Efferth

http://biopiracyconference2017.uni-mainz.de

Kiel

$$
\begin{aligned}
& \text { 6.- } 12.8 .2017 \\
& \text { ICTAM IX }
\end{aligned}
$$

International Congress on Traditional Asian

Medicines

www.ictam2017.uni-kiel.de/en

Basel/Schweiz

3.-7.9.2017

65th International Congress and Annual Meeting of the Society for Medicinal Plant and Natural Product Research (GA)

www.ga-online.org
Much, Bergisches Land

Fortbildung der GPT für Ärzte und Apotheker zu Phytopharmaka und Phytotherapie in 4 Modulen

2. Modul: 15.-17.9.2017

Die Module stehen thematisch je auch für sich allein und können einzeln gebucht werden. Wissenschaftliche Leitung: Frau Prof. Dr. Karen Nieber, Leipzig (Vorsitzende des wissenschaftlichen Kuratoriums der GPT). Organisatorische Leitung: Frau Cornelia Schwöppe (Schatzmeisterin der GPT). Die Fortbildung wird von der Landesärzte- und Landesapothekerkammer mit Fortbildungspunkten akkreditiert. www.phytotherapie.de

Francavilla al Mare/Italien 17.-20.9.2017

New and Old Phytochemicals: Their Role in Ecology, Veterinary, and Welfare

Phytochemical Society of Europe http://psecongress2017uda.wixsite.com/main

Bonn

8. Tagung für Arznei- und Gewürzpflanzenforschung

\section{6.-28.9.2017}

Universität Bonn, Veranstalter: DFA

www.dfa-aga.de

Saarbrücken

26.-29.9.2017

Jahrestagung der Deutschen Pharmazeutischen Gesellschaft

www.dphg.de

Freudenstadt

27.9.-1.10.2017

134. ZAEN-Kongress

www.zaen.org

Münster

28.-30.9.2017

Phytotherapie 2017. Wissenschaftlicher Kongress der GPT

www.phytotherapie.de

Meran/Südtirol

23.-25.10.2017

32. Südtiroler Herbstgespräche

Für Apotheker, Ärzte, Studierende

www.phytoherbst.at

Baden-Baden

1.-5.11.2017

51. Medizinische Woche Baden-Baden

www.medwoche.de 